\title{
Occurrence and characteristics of extended- spectrum $\beta$-lactamase (ESBL) producing Enterobacteriaceae in food producing animals, minced meat and raw milk
}

\author{
Nadine Geser, Roger Stephan and Herbert Hächler
}

\begin{abstract}
Background: The impact of food animals as a possible reservoir for extended-spectrum beta-lactamase (ESBL) producing Enterobacteriaceae, and the dissemination of such strains into the food production chain need to be assessed. In this study 334 fecal samples from pigs, cattle, chicken and sheep were investigated at slaughter. Additionally, 100 raw milk samples, representing bulk tank milk of 100 different dairy farms, 104 minced meat (pork and beef) samples and 67 E. coli isolates from cattle E. coli mastitis were analyzed.

Results: As many as $15.3 \%$ of the porcine, $13.7 \%$ of the bovine, $8.6 \%$ of the sheep and $63.4 \%$ of the chicken fecal samples yielded ESBL producers after an enrichment step. In contrast, none of the minced meat, none of the bulk tank milk samples and only one of the mastitis milk samples contained ESBL producing strains. Of the total of 91 isolates, 89 were E. coli, one was Citrobacter youngae and one was Enterobacter cloacae. PCR analysis revealed that 78 isolates (85.7\%) produced CTX-M group 1 ESBLs while six isolates (6.6\%) produced CTX-M group 9 enzymes. Five detected ESBLs (5.5\%) belonged to the SHV group and 2 isolates (2.2\%) contained a TEM-type enzyme. A total of 27 CTX-M producers were additionally PCR-positive for TEM-beta-lactamase. The ESBL-encoding genes of 53 isolates were sequenced of which 34 produced CTX-M-1, 6 produced CTX-M-14, 5 produced CTX-M-15 and also 5 produced SHV-12. Two isolates produced TEM-52 and one isolate expressed a novel CTX-M group 1 ESBL, CTX-M117. One isolate-aside from a CTX-M ESBL-contained an additional novel TEM-type broad-spectrum betalactamase, TEM-186.
\end{abstract}

Conclusions: The relatively high rates of ESBL producers in food animals and the high genetic diversity among these isolates are worrisome and indicate an established reservoir in farm animals.

\section{Background}

Antimicrobial resistance in bacteria has emerged as a problem in both human and veterinary medicine. One of the currently most important resistance mechanisms in Enterobacteriaceae, which reduces the efficacy even of modern expanded-spectrum cephalosporins (except cephamycins and carbapenems) and monobactams is based on plasmid-mediated production of enzymes that inactivate these compounds by hydrolyzing their $\beta$-lactam ring. Such resistance is encoded by an increasing

\footnotetext{
* Correspondence: haechlerh@fsafety.uzh.ch
Institute for Food Safety and Hygiene, Vetsuisse Faculty, University of Zurich,

* Correspondence: haechlerh@fsafety.uzh.ch CH-8057 Zurich, Switzerland
}

(c) 2012 Geser et al; licensee BioMed Central Ltd. This is an Open Access article distributed under the terms of the Creative Commons Attribution License (http://creativecommons.org/licenses/by/2.0), which permits unrestricted use, distribution, and reproduction in any medium, provided the original work is properly cited. cal broad-spectrum $\beta$-lactamases (BSBL). These variants are called extended spectrum $\beta$-lactamases (ESBL): most are derivates of TEM and SHV $\beta$-lactamase families, whereas other groups, such as CTX-M, PER and KPC $\beta$-lactamases have been described more recently [1]. The phenotypical difference between BSBLs and ESBLs is that the latter efficiently hydrolyze 3rd- and 4th-generation cephalosporins, additionally to penicillins and lower generation cephalosporins as the BSBLs are capable of. BSBLs and ESBLs are inhibited by clavulanic acid, sulbactam and tazobactam [2], a feature that is used (i) as a criterion for classification of $\beta$-lactamases and (ii) for diagnostic ESBL detection purposes. Until 
now more than 600 ESBL variants are known http:// www.lahey.org/Studies/ (last accessed January 2012). Among them, the over 100 CTX-M enzymes so far reported may be grouped into five main subgroups. Each of them is characterized by a group-representative single structure according to their amino acid sequence (group 1: CTX-M-1, group 2: CTX-M-2, group 8: CTXM-8, group 9: CTX-M-9, and group 25: CTX-M-25) [3]. As a matter of growing concern, resistance caused by ESBLs is often associated with resistance to other classes of antibiotics like fluoroquinolones, aminoglycosides and trimethoprim-sulfmethoxazole $[1,4]$.

Since the first description of ESBL producing Enterobacteriaceae isolated from hospitalized humans [5], many nosocomial outbreaks have been reported. However, since a few years, there is an increase in the detection of ESBL producing strains in the community [6]. More recently, reports have also raised concern about the dissemination of ESBL producing $E$. coli in healthy food producing animals in several countries in Europe [7-9] and Asia $[10,11]$ or in food products like meat, fish and raw milk [12-14]. Recently, Wittum et al. [15] and Doi et al. [16] described for the first time ESBL producers in healthy dairy cattle and retail meat in the USA.

Therefore, the impact of healthy farm animals as a possible reservoir for ESBL producing Enterobacteriaceae on the food processing chain has to be assessed. The aim of the present study was to screen for the occurrence of ESBL producing Enterobacteriaceae in healthy swine, cattle, sheep and chicken at slaughter as well as in milk and meat in Switzerland and to further characterize isolates.

\section{Results}

After an enrichment step ESBL producers were isolated from 90 (26.9\%) of the investigated 334 fecal samples, and one ESBL producer (1.5\%) was found in 67 E. coli mastitis milk isolates, but none was isolated from either minced meat (pork and beef) or bulk tank milk samples. The ESBL prevalence among cattle was 13.7\%, 25.3\% among calves (animals under 6 months), 8.6\% among sheep, and $15.3 \%$ among pigs. For chickens (herd level) a very high prevalence of $63.4 \%$ was determined (Table 1). All suspected isolates were phenotypically confirmed, in that they showed a synergy effect with at least 1 of 3 strips when tested with Etest-ESBL strips containing cefepime, cefotaxime or ceftazidime, and they yielded factors $>8$ when ratios of MIC (cephalosporin)/MIC (cephalosporin plus clavulanic acid) were calculated.

Almost all isolated ESBL producers were E. coli $(89$ out of 91), the exceptions being one Enterobacter cloacae isolated from a sheep, and one Citrobacter youngae isolated from a calf (Table 2).

The ESBL-encoding genes of all isolates were further characterized by PCR. A total of 78 isolates (85.7\%) produced CTX-M group 1 ESBLs while six isolates (6.6\%) produced CTX-M group 9 enzymes. Five isolates (5.5\%) were detected as producers of the SHV-ESBLs and 2 isolates (2.2\%) exclusively produced TEM-type enzymes. Twenty-seven CTX-M carriers were additionally PCRpositive for $b l a_{\mathrm{TEM}}$ genes. Of the $91 \mathrm{ESBL}$ producing isolates, 53 were selected for sequencing of the involved bla genes (Figure 1). Thirty-four isolates were CTX-M-1 producers, eight expressed additional TEM-1 and one isolate-from a pig- additionally expressed a TEM-type enzyme, TEM-186 http://www.lahey.org/Studies/, never found before (nucleotide sequence accession number JN227084). Six isolates carried CTX-M-14 with TEM-1 and five isolates specified CTX-M-15, one of which producing additional TEM-1. One isolate from a calf produced TEM-1 in combination with CTX-M-117 http://

Table 1 Occurrence of ESBL producers in food-producing animals at slaughter as well as in minced meat, bulk tank milk and isolates from bovine mastitis in Switzerland

\begin{tabular}{|c|c|c|c|}
\hline \multirow{2}{*}{$\begin{array}{l}\text { Origin } \\
\text { Cattle, fecal samples }\end{array}$} & & \multirow{2}{*}{$\begin{array}{ll}\mathbf{n} \\
124\end{array}$} & \multirow{2}{*}{$\begin{array}{l}\text { Number of samples with ESBL producers (percentage } \\
17(13.7 \% ;[95 \% \mathrm{Cl}, 8.1 ; 21.0])\end{array}$} \\
\hline & & & \\
\hline & calves & 63 & $16(25.3 \% ;[95 \% \mathrm{Cl}, 15.3 ; 37.9])$ \\
\hline & others & 61 & 1 (1.6\%; [95\% Cl, 0.4; 8.7]) \\
\hline Pig, fecal samples & & 59 & $9(15.3 \% ;[95 \% \mathrm{Cl}, 7.2 ; 26.9])$ \\
\hline Chicken, fecal samples from crates of different flocks & & 93 & 59 (63.4\%; [95\% Cl, 52.8; 73.2]) \\
\hline \multirow[t]{3}{*}{ Sheep, fecal samples } & & 58 & 5 (8.6\%; [95\% Cl, 2.9; 18.9]) \\
\hline & lambs & 40 & 2 (5.0\%; [95\% Cl, 0.6; 16.9]) \\
\hline & others & 18 & 3 (16.7\%; [95\% Cl, 3.5; 41.4]) \\
\hline Mined meat (pork, beef) & & 104 & 0 (0.0\%; [95\% Cl, 0.0; 3.4]) \\
\hline Bulk tank milk & & 100 & 0 (0.0\%; $[95 \% \mathrm{Cl}, 0.0 ; 3.6])$ \\
\hline E. coli isolates from mastitis milk & & 67 & 1 (1.5\%; $[95 \% \mathrm{Cl}, 0.3 ; 8.0])$ \\
\hline
\end{tabular}

$\mathrm{n}$ : number of samples tested

$\mathrm{Cl}$ : confidence interval 
Table 2 Identification and further characterization of the 91 ESBL producers isolated from 334 healthy food-producing animals at slaughter and from 67 Escherichia coli mastitis milk samples in Switzerland

\begin{tabular}{|c|c|c|c|c|c|c|c|c|c|c|c|c|c|c|}
\hline \multirow{2}{*}{$\begin{array}{l}\text { Sample } \\
\text { number }\end{array}$} & \multirow[t]{2}{*}{ Origin } & \multirow[t]{2}{*}{ Species } & \multirow{2}{*}{$\begin{array}{l}\text { Expressed ESBL \& accompanying } \\
\beta \text { - lactamase }\end{array}$} & \multicolumn{10}{|c|}{$\beta$-lactam antibiotic resistances } & \multirow{2}{*}{$\begin{array}{l}\text { Additional } \\
\text { resistance }\end{array}$} \\
\hline & & & & AM & AMC & $\mathrm{CF}$ & CXM & CPD & CTX & CAZ & FEP & FOX & IPM & \\
\hline 13 & pig & E. coli & CTX-M-1 & $r$ & s & $r$ & $r$ & $r$ & $i^{a}$ & $s^{a}$ & $s^{a}$ & sS & s & NA. S, SXT, TE \\
\hline 14 & pig & E. coli & CTX-M-1 & $r$ & s & $r$ & r & r & $\mathrm{i}^{\mathrm{a}}$ & $s^{a}$ & $\mathrm{i}^{\mathrm{a}}$ & s & s & NA, TE \\
\hline 64 & pig & E. coli & CTX-M-1 & $r$ & $\mathrm{~s}$ & $r$ & $r$ & $r$ & r & $s^{a}$ & $s^{a}$ & $\mathrm{~s}$ & s & SXT \\
\hline 65 & pig & E. coli & CTX-M-1 & $r$ & $\mathrm{~s}$ & $r$ & $r$ & $r$ & $i^{a}$ & $s^{a}$ & $s^{a}$ & s & s & NA, TE \\
\hline 17 & pig & E. coli & CTX-M-1 \& TEM-1 & $r$ & $s$ & $r$ & $r$ & $r$ & $r$ & $s^{a}$ & $s^{a}$ & s & $\mathrm{s}$ & CIP, NA, S, TE \\
\hline 18 & pig & E. coli & CTX-M-1 \& TEM-1 & r & s & $r$ & $r$ & $r$ & $r$ & $s^{a}$ & $i^{a}$ & $\mathrm{~s}$ & s & $\begin{array}{l}\text { C, CIP, NA, S, } \\
\text { SXT, TE }\end{array}$ \\
\hline 72 & pig & E. coli & CTX-M-1 \& TEM-1 & $r$ & s & r & $r$ & $r$ & $i^{a}$ & $s^{a}$ & $s^{a}$ & s & s & $\mathrm{S}, \mathrm{SXT}$ \\
\hline 60 & pig & E. coli & CTX-M-1 \& TEM-186 & $r$ & s & $r$ & $r$ & $r$ & $\mathrm{i}^{\mathrm{a}}$ & $s^{a}$ & $s^{a}$ & s & s & $\mathrm{S}, \mathrm{SXT}$ \\
\hline 16 & pig & E. coli & CTX-M-14 \& TEM-1 & r & $\mathrm{s}$ & r & r & $r$ & $r$ & $s^{a}$ & $s^{a}$ & $\mathrm{~s}$ & s & $\begin{array}{l}\text { CIP, GM, NA, S, } \\
\text { SXT, TE }\end{array}$ \\
\hline 46 & calf & E. coli & CTX-M-1 & r & s & r & r & $r$ & $i^{a}$ & $s^{a}$ & $s^{a}$ & s & s & C, GM, S, SXT, TE \\
\hline 112 & calf & E. coli & CTX-M-1 & $r$ & s & r & $r$ & r & $r$ & $s^{a}$ & $s^{a}$ & s & s & C, GM, S, SXT, TE \\
\hline 114 & calf & E. coli & CTX-M-1 & $r$ & $\mathrm{~s}$ & $r$ & $r$ & $r$ & $r$ & $s^{a}$ & $s^{a}$ & $\mathrm{~s}$ & $\mathrm{~s}$ & C, GM, S, SXT, TE \\
\hline 142.09_b & calf & E. coli & CTX-M-1 & $r$ & $\mathrm{~s}$ & $r$ & $r$ & $r$ & $r$ & $s^{a}$ & $s^{a}$ & $\mathrm{~s}$ & $\mathrm{~s}$ & C, GM, S, SXT, TE \\
\hline 142.09_9 & calf & $\begin{array}{l}\mathrm{C} . \\
\text { youngae }\end{array}$ & CTX-M-1 & $r$ & s & $r$ & $r$ & $r$ & $r$ & $s^{a}$ & $s^{a}$ & s & s & C, GM, S, SXT, TE \\
\hline 68 & $\begin{array}{l}\text { young } \\
\text { cow }\end{array}$ & E. coli & CTX-M-1 & $r$ & s & r & $r$ & $r$ & $r$ & $s^{a}$ & $s^{a}$ & s & s & NA \\
\hline 128 & calf & E. coli & CTX-M-1 & $r$ & $\mathrm{~s}$ & $r$ & $r$ & $r$ & $i^{a}$ & $s^{a}$ & $r$ & s & s & S, SXT, TE \\
\hline 136 & calf & E. coli & CTX-M-1 & $r$ & s & $r$ & $r$ & $r$ & $r$ & $s^{a}$ & $s^{a}$ & s & $\mathrm{s}$ & $\mathrm{CIP}, \mathrm{NA}, \mathrm{TE}$ \\
\hline 129 & calf & E. coli & CTX-M-1 \& TEM-1 & $r$ & s & $r$ & $r$ & $r$ & $\mathrm{i}^{\mathrm{a}}$ & $s^{a}$ & $s^{a}$ & s & s & $\mathrm{S}, \mathrm{SXT}, \mathrm{TE}$ \\
\hline 104 & calf & E. coli & CTX-M-1 \& TEM-1 & $r$ & $\mathrm{~s}$ & $r$ & $r$ & $r$ & $\mathrm{i}^{\mathrm{a}}$ & $s^{a}$ & $s^{a}$ & $\mathrm{~s}$ & s & C, GM, S, SXT, TE \\
\hline 47 & calf & E. coli & CTX-M-15 & $r$ & $\mathrm{~s}$ & $r$ & $r$ & $r$ & $r$ & $\mathrm{i}^{\mathrm{a}}$ & $s^{a}$ & s & s & C, GM, S, SXT, TE \\
\hline 52 & calf & E. coli & CTX-M-15 & $r$ & s & $r$ & $r$ & $r$ & $r$ & $i^{a}$ & $r$ & s & s & $\begin{array}{l}\text { CIP, NA, GM, } \\
\text { SXT, TE }\end{array}$ \\
\hline 53 & calf & E. coli & CTX-M-15 & $r$ & $\mathrm{i}$ & $r$ & $r$ & $r$ & $r$ & $i^{a}$ & $i^{a}$ & $\mathrm{~s}$ & s & $\mathrm{CIP}, \mathrm{NA}$ \\
\hline 124 & calf & E. coli & CTX-M-15 & $r$ & s & $r$ & $r$ & $r$ & $r$ & $r$ & $s^{a}$ & $\mathrm{~s}$ & s & $C I P, N A, S$ \\
\hline 142.11_n & calf & E. coli & CTX-M-117 \& TEM-1 & $r$ & $\mathrm{~s}$ & $r$ & $r$ & $r$ & $\mathrm{i}^{\mathrm{a}}$ & $s^{a}$ & $s^{a}$ & $\mathrm{~s}$ & $\mathrm{~s}$ & $\begin{array}{l}\text { C, CIP, GM, NA, } \\
\text { S, SXT, TE }\end{array}$ \\
\hline 115 & calf & E. coli & CTX-M-14 \& TEM-1 & $r$ & $\mathrm{i}$ & $r$ & $r$ & $r$ & $\mathrm{i}^{\mathrm{a}}$ & $s^{a}$ & $s^{a}$ & s & s & $C, C I P, N A, S X T$ \\
\hline 116 & calf & E. coli & CTX-M-14 \& TEM-1 & r & $\mathrm{i}$ & $r$ & $r$ & $r$ & $i^{a}$ & $s^{a}$ & $s^{a}$ & s & s & $\begin{array}{l}\text { C, CIP, GM, NA, } \\
\text { S, SXT, TE }\end{array}$ \\
\hline 2 & lamb & E. coli & CTX-M-1 \& TEM-1 & $r$ & s & $r$ & $r$ & $r$ & $r$ & $s^{a}$ & $s^{a}$ & $\mathrm{~s}$ & s & $C, N A, S, T E$ \\
\hline 108 & sheep & E. coli & CTX-M-15 \& TEM-1 & $r$ & $\mathrm{i}$ & $r$ & $r$ & $r$ & $r$ & $\mathrm{i}^{\mathrm{a}}$ & $\mathrm{i}^{\mathrm{a}}$ & $\mathrm{s}$ & s & $\begin{array}{l}\text { CIP, GM, S, SXT, } \\
\text { TE }\end{array}$ \\
\hline 100 & sheep & E. coli & CTX-M-14 \& TEM-1 & r & s & $r$ & r & $r$ & $i^{a}$ & $s^{a}$ & $s^{a}$ & s & s & $\begin{array}{l}\mathrm{C}, \mathrm{CIP}, \mathrm{GM}, \mathrm{NA} \\
\mathrm{S}, \mathrm{TE}\end{array}$ \\
\hline 102 & sheep & E. coli & CTX-M-14 \& TEM-1 & $r$ & $\mathrm{i}$ & r & r & $r$ & $\mathrm{i}^{\mathrm{a}}$ & $s^{a}$ & $s^{a}$ & s & s & $\begin{array}{l}\text { C, CIP, GM, NA, } \\
\text { S, TE }\end{array}$ \\
\hline 11 & lamb & $\begin{array}{l}\text { E. } \\
\text { cloacae }\end{array}$ & SHV-12 \& TEM-1 & r & $r$ & $r$ & $r$ & $r$ & $\mathrm{i}^{\mathrm{a}}$ & $r$ & $s^{a}$ & $r$ & s & $C, S, S X T, T E$ \\
\hline 3 & chicken & E. coli & CTX-M group $1^{+}$ & $r$ & s & $r$ & $r$ & $r$ & $i^{a}$ & $s^{a}$ & $s^{a}$ & s & $s$ & NA, TE \\
\hline 4 & chicken & E. coli & CTX-M group $1^{+}$ & r & s & $r$ & $r$ & $r$ & $r$ & $s^{a}$ & $s^{a}$ & s & $\mathrm{s}$ & $\mathrm{TE}$ \\
\hline 8 & chicken & E. coli & CTX-M group $1^{+}$ & $r$ & s & $r$ & r & $r$ & $i^{a}$ & $s^{a}$ & $s^{a}$ & s & s & SXT, TE \\
\hline 10 & chicken & E. coli & CTX-M group $1^{+}$ & $r$ & $\mathrm{~s}$ & $r$ & $r$ & $r$ & $r$ & $s^{a}$ & $s^{a}$ & $\mathrm{~s}$ & $\mathrm{~s}$ & $\mathrm{~S}, \mathrm{SXT}, \mathrm{TE}$ \\
\hline 12 & chicken & E. coli & CTX-M group $1^{+}$ & $r$ & $\mathrm{~s}$ & $r$ & $r$ & $r$ & $\mathrm{r}$ & $s^{a}$ & $s^{a}$ & $\mathrm{~s}$ & $\mathrm{~s}$ & NA, SXT, TE \\
\hline 16 & chicken & E. coli & CTX-M group $1^{+}$ & $r$ & $\mathrm{~s}$ & $r$ & $r$ & $r$ & $\mathrm{i}^{\mathrm{a}}$ & $s^{a}$ & $s^{a}$ & $s$ & s & $N A, T E$ \\
\hline
\end{tabular}


Table 2 Identification and further characterization of the 91 ESBL producers isolated from 334 healthy food-producing animals at slaughter and from 67 Escherichia coli mastitis milk samples in Switzerland (Continued)

\begin{tabular}{|c|c|c|c|c|c|c|c|c|c|c|c|c|c|c|}
\hline 17 & chicken & E. coli & CTX-M-1 & $r$ & s & $r$ & $r$ & $r$ & $r$ & $s^{a}$ & $s^{a}$ & $\mathrm{~s}$ & s & NA, TE \\
\hline 26 & chicken & E. coli & CTX-M group $1^{+}$ & $r$ & s & $r$ & $r$ & $r$ & $i^{a}$ & $s^{a}$ & $s^{a}$ & $\mathrm{~s}$ & s & $\mathrm{TE}$ \\
\hline 30 & chicken & E. coli & CTX-M group $1^{+}$ & $r$ & $\mathrm{~s}$ & $r$ & $r$ & $r$ & $\mathrm{i}^{\mathrm{a}}$ & $s^{a}$ & $s^{a}$ & $\mathrm{~s}$ & s & $\mathrm{TE}$ \\
\hline 31 & chicken & E. coli & CTX-M-1 & r & s & $r$ & r & $r$ & $i^{a}$ & $s^{a}$ & $s^{a}$ & s & s & SXT, TE \\
\hline 33 & chicken & E. coli & CTX-M group $1^{+}$ & $r$ & $\mathrm{~s}$ & $r$ & $r$ & $r$ & $r$ & $s^{a}$ & $i^{a}$ & $\mathrm{~s}$ & $\mathrm{~s}$ & NA, TE \\
\hline 35 & chicken & E. coli & CTX-M-1 & $r$ & $\mathrm{~s}$ & $r$ & $r$ & $r$ & $\mathrm{i}^{\mathrm{a}}$ & $r$ & $s^{a}$ & $\mathrm{~s}$ & s & NA, SXT, TE \\
\hline 40 & chicken & E. coli & CTX-M group $1^{+}$ & $r$ & s & $r$ & $r$ & $r$ & $i^{a}$ & $s^{a}$ & $s^{a}$ & s & s & SXT, TE \\
\hline 41 & chicken & E. coli & CTX-M group $1^{+}$ & $r$ & s & $r$ & $r$ & $r$ & $r$ & $s^{a}$ & $s^{a}$ & s & s & SXT \\
\hline 42 & chicken & E. coli & CTX-M group $1^{+}$ & r & $\mathrm{s}$ & $r$ & $r$ & $r$ & $i^{a}$ & $s^{a}$ & $s^{a}$ & s & s & NA, TE \\
\hline 43 & chicken & E. coli & CTX-M group $1^{+}$ & r & $\mathrm{s}$ & $r$ & $r$ & $r$ & $\mathrm{i}^{\mathrm{a}}$ & $s^{a}$ & $s^{a}$ & $\mathrm{~s}$ & s & SXT, TE \\
\hline$\overline{44}$ & chicken & E. coli & CTX-M group $1^{+}$ & $r$ & $\mathrm{~s}$ & $r$ & $r$ & $r$ & $\mathrm{i}^{\mathrm{a}}$ & $s^{a}$ & $s^{a}$ & $\mathrm{~s}$ & $\mathrm{~s}$ & NA, SXT, TE \\
\hline 45 & chicken & E. coli & CTX-M group $1^{+}$ & $r$ & $\mathrm{~s}$ & $r$ & $r$ & $r$ & $\mathrm{j}^{\mathrm{a}}$ & $s^{a}$ & $s^{a}$ & $\mathrm{~s}$ & $\mathrm{~s}$ & NA, SXT \\
\hline 46 & chicken & E. coli & CTX-M group $1^{+}$ & r & s & $r$ & $r$ & $r$ & $i^{a}$ & $s^{a}$ & $s^{a}$ & $\mathrm{~s}$ & s & SXT, TE \\
\hline 47 & chicken & E. coli & CTX-M-1 & $r$ & $\mathrm{~s}$ & r & $r$ & $r$ & $r$ & $s^{a}$ & $s^{a}$ & s & s & S, SXT, TE \\
\hline 48 & chicken & E. coli & CTX-M group $1^{+}$ & $r$ & $\mathrm{~s}$ & r & $r$ & $r$ & $r$ & $s^{a}$ & $s^{a}$ & s & s & SXT, TE \\
\hline 49 & chicken & E. coli & CTX-M-1 & $r$ & s & $r$ & $r$ & $r$ & $\mathrm{i}^{\mathrm{a}}$ & $s^{a}$ & $s^{a}$ & $\mathrm{~s}$ & s & S, SXT, TE \\
\hline 51 & chicken & E. coli & CTX-M group $1^{+}$ & $r$ & $\mathrm{~s}$ & $r$ & $r$ & $r$ & $\mathrm{i}^{\mathrm{a}}$ & $s^{a}$ & $s^{a}$ & $\mathrm{~s}$ & s & NA, SXT, TE \\
\hline 52 & chicken & E. coli & CTX-M group $1^{+}$ & $r$ & $\mathrm{~s}$ & $r$ & $r$ & $r$ & $r$ & $s^{a}$ & $s^{a}$ & $\mathrm{~s}$ & $\mathrm{~s}$ & NA, SXT, TE \\
\hline 53 & chicken & E. coli & CTX-M group $1^{+}$ & $r$ & $\mathrm{~s}$ & $r$ & $r$ & $r$ & $\mathrm{i}^{\mathrm{a}}$ & $s^{a}$ & $s^{a}$ & $\mathrm{~s}$ & s & SXT, TE \\
\hline 58 & chicken & E. coli & CTX-M-1 & $r$ & s & $r$ & $r$ & $r$ & $i^{a}$ & $s^{a}$ & $s^{a}$ & $\mathrm{~s}$ & s & GM, NA, SXT, TE \\
\hline 59 & chicken & E. coli & CTX-M-1 & r & s & $r$ & $r$ & $r$ & $\mathrm{i}^{\mathrm{a}}$ & $s^{a}$ & $s^{a}$ & $\mathrm{~s}$ & s & C, SXT, TE \\
\hline 60 & chicken & E. coli & CTX-M-1 & $r$ & $\mathrm{~s}$ & $r$ & $r$ & $r$ & $\mathrm{i}^{\mathrm{a}}$ & $s^{a}$ & $s^{a}$ & $\mathrm{~s}$ & s & SXT, TE \\
\hline 62 & chicken & E. coli & CTX-M group $1^{+}$ & r & $\mathrm{s}$ & $r$ & $r$ & $r$ & $\mathrm{i}^{\mathrm{a}}$ & $s^{a}$ & $s^{a}$ & $\mathrm{~s}$ & s & SXT, TE \\
\hline 63 & chicken & E. coli & CTX-M group $1^{+}$ & $r$ & $\mathrm{~s}$ & $r$ & $r$ & $r$ & $\mathrm{i}^{\mathrm{a}}$ & $s^{a}$ & $s^{a}$ & $\mathrm{~s}$ & $\mathrm{~s}$ & SXT, TE \\
\hline 64 & chicken & E. coli & CTX-M group $1^{+}$ & $r$ & $\mathrm{~s}$ & $r$ & $r$ & $r$ & $\mathrm{i}^{\mathrm{a}}$ & $s^{a}$ & $s^{a}$ & $\mathrm{~s}$ & $\mathrm{~s}$ & NA, SXT \\
\hline 65 & chicken & E. coli & CTX-M group $1^{+}$ & $r$ & $\mathrm{~s}$ & r & $r$ & $r$ & $i^{a}$ & $s^{a}$ & $s^{a}$ & $\mathrm{~s}$ & s & TE \\
\hline 67 & chicken & E. coli & CTX-M group $1^{+}$ & $r$ & s & r & r & $r$ & $\mathrm{i}^{\mathrm{a}}$ & $s^{a}$ & $s^{a}$ & $\mathrm{~s}$ & s & SXT, TE \\
\hline 68 & chicken & E. coli & CTX-M group $1^{+}$ & $r$ & s & r & r & $r$ & $i^{a}$ & $s^{a}$ & $s^{a}$ & s & s & SXT, TE \\
\hline 73 & chicken & E. coli & CTX-M group $1^{+}$ & $r$ & $\mathrm{~s}$ & $r$ & $r$ & $r$ & $\mathrm{i}^{\mathrm{a}}$ & $s^{a}$ & $s^{a}$ & $\mathrm{~s}$ & s & NA, TE \\
\hline 74 & chicken & E. coli & CTX-M-1 & $r$ & $\mathrm{~s}$ & $r$ & $r$ & $r$ & $i^{a}$ & $s^{a}$ & $s^{a}$ & $\mathrm{~s}$ & $\mathrm{~s}$ & $\overline{T E}$ \\
\hline 76 & chicken & E. coli & CTX-M group $1^{+}$ & $r$ & s & $r$ & $r$ & $r$ & $r$ & $s^{a}$ & $s^{a}$ & $\mathrm{~s}$ & $\mathrm{~s}$ & NA \\
\hline 78 & chicken & E. coli & CTX-M group $1^{+}$ & $r$ & s & $r$ & $r$ & $r$ & r & $s^{a}$ & $s^{a}$ & s & s & NA, TE \\
\hline 86 & chicken & E. coli & CTX-M-1 & $r$ & s & $r$ & $r$ & $r$ & $\mathrm{j}^{\mathrm{a}}$ & $s^{a}$ & $s^{a}$ & s & s & $\mathrm{TE}$ \\
\hline 87 & chicken & E. coli & CTX-M-1 & $r$ & $\mathrm{~s}$ & $r$ & $r$ & $r$ & $r$ & $s^{a}$ & $s^{a}$ & s & $\mathrm{s}$ & $\mathrm{TE}$ \\
\hline 88 & chicken & E. coli & CTX-M-1 & $r$ & $\mathrm{~s}$ & $r$ & $r$ & $r$ & $r$ & $s^{a}$ & $s^{a}$ & s & s & NA, SXT \\
\hline 91 & chicken & E. coli & CTX-M group $1^{+}$ & $r$ & $\mathrm{~s}$ & $r$ & $r$ & $r$ & $i^{a}$ & $s^{a}$ & $s^{a}$ & $\mathrm{~s}$ & s & NA, TE \\
\hline 92 & chicken & E. coli & CTX-M-1 & $r$ & $\mathrm{~s}$ & $r$ & $r$ & $r$ & $\mathrm{i}^{\mathrm{a}}$ & $s^{a}$ & $s^{a}$ & $\mathrm{~s}$ & $\mathrm{~s}$ & $\mathrm{~S}, \mathrm{TE}$ \\
\hline 94 & chicken & E. coli & CTX-M group $1^{+}$ & $r$ & $\mathrm{~s}$ & r & $r$ & r & $r$ & $s^{a}$ & $s^{a}$ & s & s & TE \\
\hline 96 & chicken & E. coli & CTX-M group $1^{+}$ & r & $\mathrm{s}$ & r & r & r & $i^{a}$ & $s^{a}$ & $s^{a}$ & s & s & NA, SXT, TE \\
\hline 20 & chicken & E. coli & CTX-M group 1/TEM ${ }^{+}$ & r & $\mathrm{s}$ & r & $r$ & $r$ & $\mathrm{i}^{\mathrm{a}}$ & $s^{a}$ & $s^{a}$ & s & s & CIP, NA, SXT, TE \\
\hline 27 & chicken & E. coli & CTX-M group 1/TEM ${ }^{+}$ & $r$ & $\mathrm{~s}$ & r & $r$ & $r$ & $i^{a}$ & $s^{a}$ & $s^{a}$ & s & s & S, SXT, TE \\
\hline 32 & chicken & E. coli & CTX-M-1 \& TEM-1 & $r$ & $\mathrm{~s}$ & $r$ & $r$ & $r$ & $r$ & $s^{a}$ & $s^{a}$ & $\mathrm{~s}$ & s & SXT, TE \\
\hline 36 & chicken & E. coli & CTX-M group 1/TEM ${ }^{+}$ & $r$ & $\mathrm{i}$ & $r$ & $r$ & $r$ & $i^{a}$ & $s^{a}$ & $s^{a}$ & s & s & SXT, TE \\
\hline 82 & chicken & E. coli & CTX-M group 1/TEM ${ }^{+}$ & $r$ & s & $r$ & $r$ & $r$ & r & $s^{a}$ & $s^{a}$ & $\mathrm{~s}$ & $\mathrm{~s}$ & SXT, TE \\
\hline 84 & chicken & E. coli & CTX-M-1 \& TEM-1 & r & $\mathrm{s}$ & r & $r$ & $r$ & $r$ & $s^{a}$ & $s^{a}$ & s & s & NA, SXT, TE \\
\hline 85 & chicken & E. coli & CTX-M group 1/TEM ${ }^{+}$ & $r$ & s & $r$ & $r$ & $r$ & $r$ & $s^{a}$ & $s^{a}$ & s & s & NA, SXT, TE \\
\hline
\end{tabular}


Table 2 Identification and further characterization of the 91 ESBL producers isolated from 334 healthy food-producing animals at slaughter and from 67 Escherichia coli mastitis milk samples in Switzerland (Continued)

\begin{tabular}{|c|c|c|c|c|c|c|c|c|c|c|c|c|c|c|}
\hline 97 & chicken & E. coli & CTX-M group 1/TEM ${ }^{+}$ & $r$ & $\mathrm{~s}$ & r & $r$ & $r$ & r & $s^{a}$ & $s^{a}$ & s & $\mathrm{s}$ & SXT, TE \\
\hline 5 & chicken & E. coli & SHV-12 & r & s & r & $i^{a}$ & r & $i^{a}$ & $i^{a}$ & $s^{a}$ & S & $\mathrm{s}$ & - \\
\hline 2 & chicken & E. coli & SHV-12 & r & $\mathrm{s}$ & r & $i^{a}$ & $r$ & $i^{a}$ & $s^{a}$ & $s^{a}$ & $\mathrm{~S}$ & $\mathrm{~S}$ & C, NA, TE \\
\hline 34 & chicken & E. coli & SHV-12 \& TEM-1 & r & s & r & $s^{a}$ & r & $s^{a}$ & $i^{a}$ & $s^{a}$ & s & s & NA, SXT, TE \\
\hline 77 & chicken & E. coli & SHV-12 \& TEM-1 & r & s & r & $i^{a}$ & r & $i^{a}$ & $i^{\mathrm{a}}$ & $s^{a}$ & $\mathrm{~s}$ & s & NA, SXT, TE \\
\hline 23 & chicken & E. coli & TEM-52 & r & $\mathrm{s}$ & r & $i^{a}$ & r & $i^{a}$ & $s^{a}$ & $s^{a}$ & s & $\mathrm{s}$ & NA \\
\hline 70 & chicken & E. coli & TEM-52 & r & $\mathrm{s}$ & r & r & r & $i^{a}$ & $s^{a}$ & $s^{a}$ & $\mathrm{~S}$ & $\mathrm{~S}$ & NA \\
\hline 1,006 & $\begin{array}{l}\text { mastitis } \\
\text { milk }\end{array}$ & E. coli & CTX-M-14 \& TEM-1 & r & r & r & r & r & $i^{a}$ & $\mathrm{~s}^{\mathrm{a}}$ & $s^{a}$ & $\mathrm{~s}$ & s & $\begin{array}{l}\text { C, GM, NA S, } \\
\text { SXT, TE }\end{array}$ \\
\hline
\end{tabular}

${ }^{+}$) not further characterized

$\mathrm{s}$, sensitive; i, intermediate; $r$, resistant

a) It is known that many ESBL producers may appear susceptible or intermediate to oxyimino cephalosporins in vitro if CLSI criteria are applied strictly, but do not respond to the respective therapies. Consequently, for clinical reporting these results have to be corrected to "resistant".

AM, ampicillin; AMC, amoxicillin-clavulanic acid; CF, cephalothin; CXM, cefuroxime; CPD, cefpodoxime; CTX, cefotaxime; CAZ, ceftazidime; FEP, cefepime; FOX, cefoxitin; IPM, imipenem

C, chloramphenicol; CIP, ciprofloxacin; GM, gentamicin; NA, nalidixic acid; S, streptomycin; SXT, trimethoprim-sulfamethoxazole; TE, tetracycline

www.lahey.org/Studies/, a novel CTX-M group 1 ESBL with an amino acid sequence never found before (nucleotide sequence accession number JN227085). Finally, two TEM-52 ESBL producers, and 5 SHV-12 carriers were found, three of the latter featuring additional TEM-1 (Table 2).

Besides the $\beta$-lactam resistances, the isolates were also tested for resistance to other classes of antibiotics. We found 76 (cattle: 13/17, pig: 6/9, sheep: 5/5, chicken: 51/ 62, milk: $1 / 1$ ) out of 91 isolates resistant to tetracycline (83.5\%), 59 isolates (cattle: 13/17, pig: 6/9, sheep: $2 / 5$, chicken: $37 / 62$, milk: $1 / 1$ ) resistant to trimethoprim-sulfamethoxazole (64.8\%), 43 isolates (cattle: $8 / 17$, pig: 6/9, sheep: 4/5, chicken: $24 / 62$, milk: $1 / 1$ ) resistant to nalidixic acid (47.3\%) and 31 (cattle: 13/17, pig: 6/9, sheep:

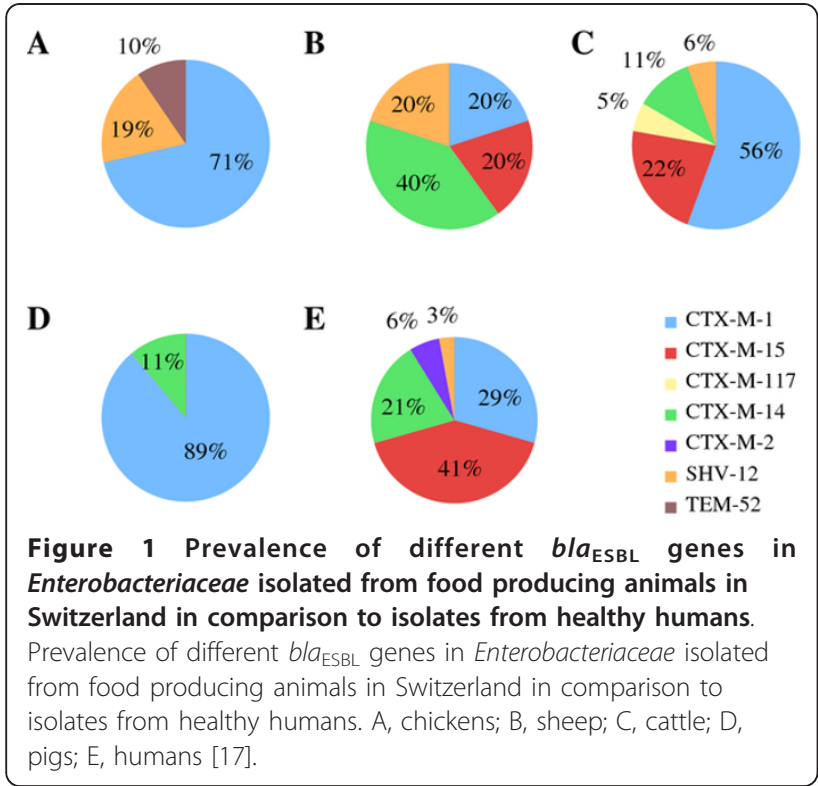

5/5, chicken: 6/62, milk: $1 / 1)$ resistant to at least one aminoglycoside (34.0\%). Furthermore, 20 isolates (cattle: 11/17, pig: $2 / 9$, sheep: $4 / 5$, chicken: $2 / 62$, milk: $1 / 1$ ) showed resistance against chloramphenicol (22.0\%), and 18 isolates (cattle: $7 / 17$, pig: $3 / 9$, sheep: $3 / 5$, chicken: $4 /$ 62, milk: 1/1) showed resistance against ciprofloxacin (19.8\%). One isolate from chicken faeces showed only resistance to ß-lactam-antibiotics, and none of the tested isolates was resistant to imipenem (Table 2).

\section{Discussion}

Recently, an increase in studies, carried out in different countries, and describing the prevalence and characteristics of ESBL producing Enterobacteriaceae in cattle for example $[2,15,18,19]$ and in pigs and chicken for example [20-25] were published. Moreover, some studies describing ESBL producing Enterobacteriaceae in salads [6], in meat [13,14] and in raw milk [12] are available. Since there seem to be geographical variations in the occurrence of different ESBL variants (e.g. CTX-M-9 in Spain as opposed to CTX-M group 1 in the Northern European countries [26]), it is therefore important to have a detailed overview based on geographical distribution and this knowledge was so far limited in Switzerland. Therefore, the present study provides further data concerning healthy animals (among them about sheep for the first time in literature), minced meat and bulk tank milk samples in Switzerland.

The high ESBL occurrence determined for all investigated animals in this study is surprising, given the fact that Switzerland is a country with a strict policy of antibiotic use [27]. Nevertheless, one reason could be the use of $\beta$-lactams-and even 4th generation cephalosporins-in veterinary medicine $[28,29]$. Another reason could be co-selection of multiple resistance mechanisms 
through the use of various antibiotics, due to the fact that resistance genes for aminoglycosides, tetracycline and trimethoprim-sulfametoxazole are frequently placed on single conjugative plasmids, as is often also the case with bla $a_{\mathrm{ESBL}}$ genes $[4,26]$. For food producing animals very limited data on the occurrence of ESBL producing Enterobacteriaceae had been available before in Switzerland, but there is a study about CTX-M producers in Swiss patients [30], and a recent report about occurrence of ESBL carriers in the healthy Swiss human population $(5.8 \%)$ [17]. This is lower than the rates from food animals (8.6\% to $63.4 \%$ ) presented in this study. These frequencies primarily imply a reservoir of ESBL producers in farm animals. Supporting this view, a study from the Netherlands described the same CTX-M-type in chicken meat and humans [31]. In Switzerland, these findings cannot be confirmed because of the predominance of CTX-M-1 in animals and the predominance of CTX-M-15 in humans $[17,30]$. The predominance of CTX-M group 1 enzymes and the rare prevalence of CTX-M group 9, as seen in our study, has also recently been described in strains from healthy food-producing animals in Denmark, Portugal and France [8,32,33]. All of the TEM enzymes co-expressed alongside with CTXM ESBLs were broad-spectrum $\beta$-lactamases-mostly TEM-1-conveying no ESBL phenotype. In contrast, two strains, expressing TEM enzymes exclusively, featured the TEM-ESBL TEM-52 (Table 2). In other countries TEM-ESBLs are much more frequently found in animals, especially in chickens [21,34].

Given the relatively high occurrence of ESBL producers in fecal samples from animals in our study, it is striking, that no ESBL producers could be found in either bulk tank milk or beef and pork minced meat. We hypothesise that the very high hygiene standards for slaughtering together with the selection of the raw meat for minced meat production and the quality based prizing system of bulk tank milk in Switzerland could be the reason for this favourable situation.

\section{Conclusion}

The occurrence of ESBL producing Enterobacteriaceae in the fecal microflora of farm animals represents an obvious risk for contamination of raw food products from animal origin. However, since no ESBL producers were found in the examined food samples and our data concerning the ESBL type distribution in animals compared to healthy human carriers do not correlate well, animal food products can hardly be the major vector for ESLB carriage in the human population in Switzerland. Nevertheless, due to the generally high ESBL occurrence in food animals in Switzerland prudent use of antibiotics in veterinary medicine and strict hygiene measures during slaughtering and milking still remain important.
Finally, on the basis of the massive CTX-M-1 predominance in animals in our study compared to its relatively low frequency in healthy humans, further investigational efforts into the origin of the unexplained high occurrence of CTX-M-15 in the human population are warrented.

\section{Methods \\ Sampling}

Fecal samples were collected in October 2009 and from November 2010 to March 2011 from 334 healthy foodproducing animals at slaughter in Switzerland: 59 pigs (57 fattening pigs, 2 piglets), 124 cattle (63 calves, 26 young cows, 18 fattening bulls, 10 cows and 7 bullocks) and 58 sheep (40 lambs, 18 sheep older than one year). To prevent sample clustering, at most two samples per farm were taken. The farms are distributed throughout Switzerland (16 cantons). Sampling was done with one swab per animal at a big EU-approved slaughterhouse (on average 1,000 pigs, 800 cattle, 60 sheep per day). Furthermore, 93 fecal samples of chicken were collected at the entry of a big EU-approved poultry slaughterhouse (on average 50,000 animals per day) from the crates of 93 poultry flocks (approximately 6,000 chicken per flock) distributed throughout Switzerland (14 cantons). Afterwards the swabs were put into an empty sterile tube, transported to the lab and processed within 6 hours of collection.

A total of 104 fresh minced meat samples (55 beef, 15 pork, 9 beef/pork, 3 veal, 3 beef/veal/pork, 2 lamb, 2 beef/lamb, and 15 of unknown origin) collected at 20 different days from a big meat processing plant $(67.3 \%$ of the samples), which is supplying minced meat to retail stores and covers about $50 \%$ of the Swiss market and from local butcher shops (32.7\% of the samples) were investigated.

Finally, 100 raw milk samples, representing bulk tank milk of 100 different dairy farms, were collected in April 2011 at a big dairy manufacturing plant in Switzerland. Furthermore, 67 E. coli isolates from cattle E. coli mastitis milk were investigated.

\section{Microbiological analysis}

About $1 \mathrm{~g}$ of each fecal sample was enriched in $10 \mathrm{ml}$ EE broth (BD, Franklin Lakes, USA) for 24 hours at $37^{\circ}$ C. Moreover, $10 \mathrm{ml}$ of the milk or $10 \mathrm{~g}$ of the meat samples were enriched for 24 hours at $37^{\circ} \mathrm{C}$ in $100 \mathrm{ml}$ of EE Broth. Thereafter the enrichment was streaken onto Brilliance ESBL agar (Oxoid, Hampshire, UK), which was incubated at $37^{\circ} \mathrm{C}$ for 24 hours under aerobic conditions. All grown colonies of different color and/or morphology were selected and subcultured onto triple sugar iron (TSI) agar (BD, Franklin Lakes, USA) at $37^{\circ} \mathrm{C}$ for 24 hours. By the oxidase test, nonfermenters were 
discarded, and oxidase-negative colonies were subjected to identification by API ID 32 E (bioMérieux, Marcy 1'Etoile, France). Some isolates, yielding doubtful results, were subjected to genetic identification based on sequencing of $16 \mathrm{~S}$ rRNA and rpoB gene fragments [35].

\section{Antimicrobial susceptibility testing and ESBL detection}

All isolates were subjected to susceptibility testing against 17 antimicrobial agents by the disc diffusion method according to CLSI protocols and the results were evaluated according to CLSI criteria [36]. The antibiotics (Becton Dickinson, Sparks, MD USA) tested were: ampicillin (AM), amoxicillin/clavulanic acid (AMC), cephalothin $(\mathrm{CF})$, cefuroxime (CXM), cefpodoxime (CPD), cefotaxime (CTX), ceftazidime (CAZ), cefepime (FEP), cefoxitin (FOX), imipenem (IMP), chloramphenicol (C), ciprofloxacin (CIP), gentamicin (GM), nalidixic acid (NA), streptomycin (S), trimethoprim-sulfamethoxazole (SXT), tetracycline (TE). The $A M C$ disc was placed between those containing CPD and CAZ, and the resulting synergy effects were documented. The isolates, which showed a synergy effect between AMC and CPD and/or AMC and CAZ, were then confirmed as ESBL producers on Muller-Hinton agar plates using E-Test-ESBL strips containing cefotaxime, cefepime or ceftazidime each alone and in combination with clavulanic acid (bioMérieux, Marcy 1'Etoile, France) according to the manufacturer's recommendations.

\section{Characterization of $\beta$-lactamases}

Bacterial isolates confirmed for producing ESBLs were further analysed by PCR. DNA was extracted by a standard heat lysis protocol. Thereafter, five specific primer sets (custom-synthesized by Microsynth, Balgach, Switzerland) were used to search for $b l a_{\mathrm{TEM}}, b l a_{\mathrm{SHV}}$ and $b l a_{\text {CTX-M }}$ genes [37-39].

\section{PCR amplification and sequencing of bla open reading frames (ORF)}

The ESBL-encoding genes of the isolated ESBL producers from cattle (17), sheep (5), pigs (9) and mastitis milk, as well as of 21 of the 59 isolates from chickens were sequenced. To be able to sequence the whole ORFs, five PCR/sequencing primers were used. Two per bla family were the respective screening primers (see above), three per bla family were designed newly (this study), and custom-synthesized by Microsynth (Balgach, Switzerland). Primers were: primer $1_{1}$ forward 5'-AAACACACGTGGAATTTAGGG-3' primer $22_{1}$ forward, 5'AAAAATCACTGCGCCAGTTC-3' [39], primer $3_{1}$ reverse, 5'-AGCTTATTCATCGCCACGTT-3' [39], primer $4_{1}$ reverse, 5'-CCGTCGGTGACGATTTTAGCC-3', primer $5_{1}$ reverse, 5'-CCGATGACTATGCGCAC
TGGG-3', for bla $a_{\text {CTX-M-group } 1} ; 1_{2}$ forward, 5'-TTT TGCCGTACCTGCGTACCC-3', primer 2 forward, 5'CGACGCTACCCCTGCTATT-3' [39], primer $3_{2}$ reverse, 5-CCAGCGTCAGATTTTTCAGG-3' [39], $4_{2}$ reverse, 5'-CCGTGGGTTACGATTTTCGCC-3', $5_{2}$ reverse, 5'-TTGGTCCAGAAAAAAGAGCGG-3' for bla $a_{\mathrm{CTX} \text {-M-group } 2}$ and $1_{9}$ forward, 5'-TGATGTAACACG GATTGACCG-3' 2 , forward, 5'-CAAAGAGAGTGCA ACGGATG-3' [39], 3, reverse, 5'-ATTGGAAAGCGTT CATCACC-3' [39], $4_{9}$ reverse, 5-AAACCAGTTA CAGCCCTTCGG-3' and 59 reverse, 5-TGGAGC CACGgTtGatgagGG-3' for bla $a_{\mathrm{CTX} \text {-M-group9. }}$ Primer pairs for PCR were: 1-3, 1-4, 1-5, 2-4 and 2-5 and PCR-conditions comprised initial denaturation at $94^{\circ} \mathrm{C}$ for $15 \mathrm{sec}$, followed by 35 cycles each including steps for denaturation at $94^{\circ} \mathrm{C}$ for $30 \mathrm{sec}$, annealing at $53^{\circ} \mathrm{C}$ for $30 \mathrm{sec}$ and elongation at $72^{\circ} \mathrm{C}$ for $30 \mathrm{sec}$, followed by a final extension at $72^{\circ} \mathrm{C}$ for $7 \mathrm{~min}$. For sequencing of TEM genes the same primers and PCR conditions as before were used [38], whereas for the SHV genes sequencing primers were used as described previously [40]. PCR-conditions for $b l a_{\mathrm{SHV}}$ genes were the same as those for TEM genes [38]. Resulting amplicons were purified using the PCR Purification Kit (QIAGEN, Courtaboeuf, France) according to the manufacturer's recommendations. Custom-sequencing was performed at Microsynth (Balgach, Switzerland) and the nucleotide and protein sequences were analyzed with Codon Code Aligner V. 3.7.1.1. For database searches NCBI at the BLASTN program package http://www.ncbi.nlm.nih.gov/ blast/ was used.

\section{Statistical analysis}

95 percent confidence intervals were calculated using $\mathrm{R}$ software http://www.R-project.org/.

\section{Acknowledgements}

We thank Peter Kuhnert, Vetsuisse Faculty University of Berne, for his support with species identification, Grethe Sägesser, National Centre for Enteropathogenic Bacteria and Listeria, Vetsuisse Faculty University of Zurich, for her support concerning antibiotic testing, and the Swiss Federal Office of Public Health and the Swiss Federal Veterinary Office for partial financial support.

\section{Authors' contributions}

$\mathrm{NG}$ and $\mathrm{HH}$ were responsible for isolation and characterization of strains and drafted the manuscript. $\mathrm{RS}$ and $\mathrm{HH}$ designed the study and edited the manuscript. All authors read, commented on, and approved the final manuscript.

Received: 21 September 2011 Accepted: 7 March 2012

Published: 7 March 2012

\section{References}

1. Coque TM, Baquero F, Cantòn R: Increasing prevalence of ESBL-producing Enterobacteriaceae in Europe. Eurosurveill 2008, 13, pii: 19044.

2. Bradford PA, Petersen PJ, Fingerman IM, White DG: Characterization of expanded-spectrum cephalosporin resistance in $E$. coli isolates 
associated with bovine calf diarrhoeal disease. J Antimicrob Chemother 1999, 44:607-610.

3. Bonnet R: Growing group of extended-spectrum $\beta$-lactamases: the CTXM enzymes. Antimicrob Agents Chemother 2004, 48:1-14.

4. Gniadkowski M: Evolution and epidemiology of extended-spectrum betalactamases (ESBLs) and ESBL-producing microorganisms. Clin Microbiol Infect 2001, 7:597-608.

5. Knothe $H$, Shah $P$, Krcmery $V$, Antal M, Mitsuhahi S: Transferable resistance to cefotaxime, cefoxitin, cefamamdole and defuroxime in clinical isolates of Klebsiella pneumoniae and Serratia marcescens. Infect 1983, 11:315-317.

6. Mesa RJ, Blanc V, Blanch AR, Cortés P, González JJ, Lavilla S, Miró E, Muniesa M, Saco M, Tórtola MT, Mirelis B, Coll P, Llagostera M, Prats G, Navarro FJ: Extended-spectrum b-lactamase-producing

Enterobacteriaceae in different environments (humans, food, animal farms and sewage). Antimicrob Chemother 2006, 58:211-215.

7. Cortés P, Blanc V, Mora A, Dahbi G, Blanco JE, Blanco M, López C, Andreu A, Navarro F, Alonso MP, Bou G, Blanco J, Llagostera M: Isolation and characterization of potentially pathogenic antimicrobial-resistant Escherichia coli strains from chicken and pig farms in Spain. Appl Environ Microbiol 2010, 76:2799-2805.

8. Gonçalves A, Torres C, Silva N, Carneiro C, Radhouani H, Coelho C, Araújo C, Rodrigues J, Vinué L, Somalo S, Poeta P, Igrejas G: Genetic characterization of extended-spectrum $\beta$-Lactamases in Escherichia coli isolates of pigs from a Portuguese intensive swine farm. Foodborne Pathog Dis 2010, 7:1569-1573.

9. Meunier D, Jouy E, Lazizzera C, Kobisc M, Madec JY: CTX-M-1- and CTX-M15-type b-lactamases in clinical Escherichia coli isolates recovered from food-producing animals in France. Int J Antimicrob Agents 2006, 28:402-407.

10. Duan RS, Sit TH, Wong SS, Wong RC, Chow KH, Mak GC, Yam WC, Ng LT, Yuen KY, Ho PL: Escherichia coli producing CTX-M $\beta$-lactamases in food animals in Hong Kong. Microb Drug Resist 2006, 12:145-148.

11. Tian GB, Wang HN, Zou LK, Tang JN, Zhao YW, Ye MY, Tang JY, Zhang Y, Zhang AY, Yang X, Xu CW, Fu YJ: Detection of CTX-M-15, CTX-M-22, and SHV-2 extended-spectrum $\beta$-lactamases (ESBLs) in Escherichia coli fecalsample isolates from pig farms in China. Foodborne Pathog Dis 2009, 6:297-304

12. Hammad AM, Ahmed AM, Ishida $Y$, Shimamoto T: First characterization and emergence of SHV-60 in raw milk of a healthy cow in Japan. J Vet Med Sci 2008, 70:1269-1272.

13. Jensen $L B$, Hasman $H$, Agers $\varnothing$ Y, Emborg HD, Aarestrup FM: First description of an oxyimino-cephalosporin-resistant, ESBL-carrying Escherichia coli isolated from meat sold in Denmark. J Antimicrob Chemother 2006, 57:793-794.

14. Jouini A, Vinué L, Slama KB, Sáenz Y, Klibi N, Hammami S, Boudabous A Torres C: Characterization of CTX-M and SHV extended-spectrum blactamases and associated resistance genes in Escherichia coli strains of food samples in Tunisia. J Antimicrob Chemother 2007, 60:1137-1141.

15. Wittum TE, Mollenkopf DF, Daniels JB, Parkinson AE, Mathews UL, Fry PR, Abley MJ, Gebreyes WA: CTX-M-type expended-spectrum $\beta$-lactamases present in Escherichia coli from the feces of cattle in Ohio, United States. Foodborne Path Dis 2010, 7:1575-1579.

16. Doi Y, Paterson DL, Egea P, Pascual A, Lopez-Cerero L, Navarro MD, AdamsHaduch JM, Qureshi ZH, Sidjabat HE, Rodriguez-Bano J: Extended-spectrum and CMY-type beta-lactamase-producing Escherichia coli in clinical samples and retail meat from Pittsburgh, USA and Seville, Spain. Clin Microbiol Infect 2010, 16:33-38.

17. Geser N, Stephan R, Korczak BM, Beutin L, Hächler H: Molecular identification of blaESBL genes from Enterobacteriaceae isolated from healthy human carriers in Switzerland. Antimicrob Agents Chemother 2012, 56:1609-1612.

18. Horton RA, Randall LP, Snary EL, Cockrem H, Lotz S, Wearing H, Duncan D, Rabie A, McLaren I, Watson E, La Ragione RM, Coldham NG: Fecal carriage and shedding density of CTX-M extended-spectrum ß-lactamaseproducing Escherichia coli in cattle, chickens, and pigs: Implications for environmental contamination and food production. Appl Environ Microbiol 2011, 77:3715-3719.

19. Madec JY, Lazizzera C, Châtre P, Martin S, Lepage G, Ménard MF, Lebreton P, Rambaud T, Meunier D: Prevalence of fecal carriage of acquired expanded-spectrum cephalosporin resistance in
Enterobacteriaceae strains from cattle in France. J Clin Microbiol 2008, 46:1566-1567.

20. Blanc V, Mesa R, Saco M: ESBL- and plasmidic class C $\beta$-lactamaseproducing $\mathrm{E}$. coli strains isolated from poultry, pig and rabbit farms. Vet Microbiol 2006, 118:299-21.

21. Costa D, Vinué L, Poeta $P$, Coelho AC, Matos M, Sáenz $Y$, Somalo $S$, Zarazaga M, Rodrigues J, Torres C: Prevalence of extended-spectrum betalactamase-producing Escherichia coli isolates in fecal samples of broilers. Vet Microbiol 2009, 138:339-344.

22. Dierkx C, van Essen-Zandbergen A, Veldman K, Smith H, Mevius D: Increased detection of extended spectrum beta-lactamase producing Salmonella enterica and Escherichia coli isolates from poultry. Vet Microbiol 2010, 145:273-278.

23. Kojima A, Ishii Y, Ishihara K, Esaki H, Asai T, Oda C, Tamura Y, Takahashi T, Yamaguchi K: Extended-spectrum-betalactamase-producing Escherichia coli strains isolated from farm animals from 1999 to 2002: report from the Japanese veterinary antimicrobial resistance monitoring program. Antimicrob Agents Chemother 2005, 49:3533-3537.

24. Moreno MA, Teshager T, Porrero C, García M, Escudero E, Torres C, Domínguez L: Abundance and phenotypic diversity of Escherichia coli isolates with diminished susceptibility to expanded-spectrum cephalosporins in faeces from healthy food animals after slaughter. Vet Microbiol 2007, 120:363-369.

25. Riaño I, Moreno MA, Teshager T, Sáenz Y, Domínguez L, Torres C: Detection and characterization of extended-spectrum betalactamases in Salmonella enterica strains of healthy food animals in Spain. J Antimicrob Chemother 2006, 58:844-847.

26. Cantòn R, Coque TM: The CTX-M $\beta$-lactamase pandemic. Curr Opin Microbiol 2006, 9:466-475.

27. Filippini M, Masiero G, Moschetti K: Socioeconomic determinants of regional differences in outpatient antibiotic consumption: evidence from Switzerland. Health Policy 2006, 78:77-92.

28. Cavaco LM, Abatih, Aarestrup FM, Guardabassi L: Selection and persistence of CTX-M-producing Escherichia coli in the intestinal flora of pigs treated with amoxicillin, ceftiofur or cefquinome. Antimicrob Agents Chemother 2008, 52:3612-3616.

29. Medeiros AA: Evolution and dissemination of beta-lactamases accelerated by generations of beta-lactam antibiotics. Clin Infect Dis 1997, 24:19-45.

30. Lartigue MF, Zinsius C, Wenger A, Bille J, Poirel L, Nordmann P: Extendedspectrum $\beta$-lactamases of the CTX-M type now in Switzerland. Antimicrob Agents Chemother 2007, 51:2855-2860.

31. Overdevest I, Willemsen I, Rijnsburger M, Eustace A, Xu L, Hawkey P, Heck M, Savelkoul P, Vandenbroucke-Grauls C, van der Zwaluw K, Huijsdens $X$, Kluytmans J: Extended-spectrum ß-lactamase genes of Escherichia coli in chicken meat and humans, the Netherlands. Emerg Infect Dis 2011, 17:1216-1222.

32. Aarestrup FM, Hasman H, Agerso Y, Jensen LB, Harksen S, Svensmark B: First description of blaCTX-M-1-carrying Escherichia coli isolates in Danish primary food production. J Antimicrob Chemother 2006, 57:1258-1259.

33. Girlich D, Poirel L, Carattoli A, Kempf I, Lartique MF, Bertini A, Nordmann P. Extended-spectrum (beta)-lactamase CTX-M-1 in Escherichia coli in healthy poultry in France. Appl Environ Microbiol 2007, 73:4681-4685.

34. Smet A, Martel A, Persoons D, Dewulf J, Heyndricks M, Catry B, Herman L, Haesebrouck F, Butaye P: Diversity of extended- spectrum $\beta$-lactamases and class $C \beta$ - lactamases among cloacal Escherichia coli isolates in Belgian broiler farms. Antimicrob Agents Chemother 2008, 52:1238-1243.

35. Korczak B, Christensen H, Emler S, Frey J, Kuhnert P: Phylogeny of the family Pasteurellaceae based on $r p o B$ sequences. Int J Syst Evol Microbiol 2004, 54:1393-1399.

36. Clinical and Laboratory Standards Institute: Performance Standards for Antimicrobial Susceptibility Testing; Eighteenth Informational Supplement. CLSI document M100-S18 Wayne; 2008

37. Geser N, Stephan R, Kuhnert P, Zbinden R, Käppeli U, Cernela N, Hächler H: Fecal carriage of extended-spectrum ß-lactamase-producing Enterobacteriaceae in swine and cattle at slaughter in Switzerland. J Food Prot 2011, 74:446-449.

38. Pitout JD, Thomson KS, Hanson ND, Erhardt AF, Moland ES, Sanders CC: Beta-lactamases responsible for resistance to expanded-spectrum cephalosporins in Klebsiella pneumoniae, Escherichia coli, and Proteus 
mirabili isolates recovered in South Africa. Antimicrob Agents Chemother 1998, 42:1350-1354.

39. Woodford N, Fagan EJ, Ellington MJ: Multiplex PCR for rapid detection of genes encoding CTX-M extended-spectrum $\beta$-lactamases. J Antimicrob Chemother 2005, 57:154-155.

40. Nüesch-Inderbinen MT, Hächler H, Kayser FH: Detection of genes coding for extended-spectrum SHV beta-lactamases in clinical isolates by a molecular genetic method, and comparison with the E test. Eur J Clin Microbiol Infect Dis 1996, 15:398-402.

doi:10.1186/1746-6148-8-21

Cite this article as: Geser et al: Occurrence and characteristics of extended-spectrum $\beta$-lactamase (ESBL) producing Enterobacteriaceae in food producing animals, minced meat and raw milk. BMC Veterinary Research 2012 8:21.

Submit your next manuscript to BioMed Central and take full advantage of:

- Convenient online submission

- Thorough peer review

- No space constraints or color figure charges

- Immediate publication on acceptance

- Inclusion in PubMed, CAS, Scopus and Google Scholar

- Research which is freely available for redistribution

Submit your manuscript at www.biomedcentral.com/submit 